\title{
REVIEWS
}

EXPERT CONSENSUS DOCUMENT

\section{Third universal definition of myocardial infarction}

Kristian Thygesen, Joseph S. Alpert, Allan S. Jaffe, Maarten L. Simoons, Bernard R. Chaitman

and Harvey D. White: the Writing Group on behalf of the Joint ESC/ACCF/AHA/WHF Task Force for the Universal Definition of Myocardial Infarction

Task Force members | Chairpersons: Kristian Thygesen (Denmark), Joseph S. Alpert (USA), Harvey D. White (New Zealand). Biomarker Subcommittee: Allan S. Jaffe (USA), Hugo A. Katus (Germany), Fred S. Apple (USA), Bertil Lindahl (Sweden), David A. Morrow (USA). ECG Subcommittee: Bernard R. Chaitman (USA), Peter M. Clemmensen (Denmark), Per Johanson (Sweden), Hanoch Hod (Israel). Imaging Subcommittee: Richard Underwood (UK), Jeroen J. Bax (The Netherlands), Robert O. Bonow (USA), Fausto Pinto (Portugal), Raymond J. Gibbons (USA). Classification Subcommittee: Keith A. Fox (UK), Dan Atar (Norway), L. Kristin Newby (USA), Marcello Galvani (Italy), Christian W. Hamm (Germany). Intervention Subcommittee: Barry F. Uretsky (USA), Ph. Gabriel Steg (France), William Wijns (Belgium), Jean-Pierre Bassand (France), Phillippe Menasché (France), Jan Ravkilde (Denmark). Trials \& Registries Subcommittee: E. Magnus Ohman (USA), Elliott M. Antman (USA), Lars C. Wallentin (Sweden), Paul W. Armstrong (Canada), Maarten L. Simoons (The Netherlands). Heart Failure Subcommittee: James L. Januzzi (USA), Markku S. Nieminen (Finland), Mihai Gheorghiade (USA), Gerasimos Filippatos (Greece). Epidemiology Subcommittee: Russell V. Luepker (USA), Stephen P. Fortmann (USA), Wayne D. Rosamond (USA), Dan Levy (USA), David Wood (UK). Global Perspective Subcommittee: Sidney C. Smith (USA), Dayi Hu (China), José-Luis Lopez-Sendon (Spain), Rose Marie Robertson (USA), Douglas Weaver (USA), Michal Tendera (Poland), Alfred A. Bove (USA), Alexander N. Parkhomenko (Ukraine), Elena J. Vasilieva (Russia), Shanti Mendis (Switzerland).

Thygesen, K. et al. Nat. Rev. Cardiol. 9, 620-633 (2012); published online 25 August 2012; doi:10.1038/nrcardio.2012.122

\section{Introduction}

Myocardial infarction (MI) can be recognized by clinical features, including electrocardiographic (ECG) findings, elevated values of biochemical markers (biomarkers) of myocardial necrosis, and by imaging, or may be defined by pathology (Box 1). It is a major cause of death and disability worldwide. MI may be the first manifestation of coronary artery disease (CAD) or it may occur, repeatedly, in patients with established disease. Information on MI rates can provide useful information regarding the

\footnotetext{
Competing interests

The members of the Task Force of the ESC, the ACCF, the AHA and the WHF have participated independently in the preparation of this document, drawing on their academic and clinical experience and applying an objective and clinical examination of all available literature. Most have undertaken - and are undertaking - work in collaboration with industry and governmental or private health providers (research studies, teaching conferences, consultation), but all believe such activities have not influenced their judgment. The best guarantee of their independence is in the quality of their past and current scientific work. However, to ensure openness, their relationships with industry, government and private health providers are reported as supplementary information online (www.nature.com/nrcardio). Expenses for the Task Force/ Writing Committee and preparation of this document were provided entirely by the above-mentioned joint associations.
}

burden of CAD within and across populations, especially if standardized data are collected in a manner that distinguishes between incident and recurrent events. From the epidemiological point of view, the incidence of MI in a population can be used as a proxy for the prevalence of CAD in that population. The term 'myocardial infarction' may have major psychological and legal implications for the individual and society. It is an indicator of one of the leading health problems in the world and it is an outcome measure in clinical trials, observational studies and quality assurance programs. These studies and programs require a precise and consistent definition of MI.

In the past, a general consensus existed for the clinical syndrome designated as MI. In studies of disease prevalence, the World Health Organization (WHO) defined MI from symptoms, ECG abnormalities and cardiac enzymes. However, the development of ever more sensitive and myocardial tissue-specific cardiac biomarkers and more sensitive imaging techniques now allows for detection of very small amounts of myocardial injury or necrosis. Additionally, the management of patients with MI has significantly improved, resulting in less myocardial injury and necrosis, in spite of a similar clinical presentation. Moreover, it appears necessary 
to distinguish the various conditions which may cause MI, such as 'spontaneous' and 'procedure-related' MI. Accordingly, physicians, other health care providers and patients require an up-to-date definition of MI.

In 2000, the First Global MI Task Force presented a new definition of MI, which implied that any necrosis in the setting of myocardial ischemia should be labeled as MI. ${ }^{1}$ These principles were further refined by the Second Global MI Task Force, leading to the Universal Definition of Myocardial Infarction Consensus Document in 2007, which emphasized the different conditions which might lead to an MI. ${ }^{2}$ This document, endorsed by the European Society of Cardiology (ESC), the American College of Cardiology Foundation (ACCF), the American Heart Association (AHA), and the World Heart Federation (WHF), has been well accepted by the medical community and adopted by the WHO. ${ }^{3}$ However, the development of even more sensitive assays for markers of myocardial necrosis mandates further revision, particularly when such necrosis occurs in the setting of the critically ill, after percutaneous coronary procedures or after cardiac surgery. The Third Global MI Task Force has continued the Joint ESC/ACCF/AHA/WHF efforts by integrating these insights and new data into the current document, which now recognizes that very small amounts of myocardial injury or necrosis can be detected by biochemical markers and/or imaging.

\section{Pathological characteristics of myocardial ischemia and infarction}

MI is defined in pathology as myocardial cell death due to prolonged ischemia. After the onset of myocardial ischemia, histological cell death is not immediate, but takes a finite period of time to develop-as little as $20 \mathrm{~min}$, or less in some animal models. ${ }^{4}$ It takes several hours before myocardial necrosis can be identified by macroscopic or microscopic post-mortem examination. Complete necrosis of myocardial cells at risk requires at least $2-4 \mathrm{~h}$, or longer, depending on the presence of collateral circulation to the ischemic zone, persistent or intermittent coronary arterial occlusion, the sensitivity of the myocytes to ischemia, preconditioning, and individual demand for oxygen and nutrients. ${ }^{2}$ The entire process leading to a healed infarction usually takes at least 5-6 weeks. Reperfusion may alter the macroscopic and microscopic appearance.

\section{Biomarker detection of myocardial injury with necrosis}

Myocardial injury is detected when blood levels of sensitive and specific biomarkers such as cardiac troponin (cTn) or the MB fraction of creatine kinase (CKMB) are increased. ${ }^{2}$ Cardiac troponin I and $\mathrm{T}$ are components of the contractile apparatus of myocardial cells and are expressed almost exclusively in the heart. Although elevations of these biomarkers in the blood reflect injury leading to necrosis of myocardial cells, they do not indicate the underlying mechanism. ${ }^{5}$ Various possibilities have been suggested for release of structural proteins from the myocardium, including normal turnover of myocardial cells, apoptosis, cellular release of troponin

\section{Box 1 | Definition of myocardial infarction}

\section{Criteria for acute myocardial infarction}

The term acute myocardial infarction should be used when there is evidence of myocardial necrosis in a clinical setting consistent with acute myocardial ischemia. Under these conditions, any one of the following criteria meets the diagnosis for myocardial infarction:

- Detection of a rise and/or fall of cardiac biomarker values (preferably cardiac troponin) with at least one value above the $99^{\text {th }}$ percentile URL and with at least one of the following: (i) symptoms of ischemia, or (ii) new or presumed new significant ST-segment-T wave (ST-T) changes or new left bundle branch block, or (iii) development of pathological Q waves in the electrocardiogram, or (iv) imaging evidence of new loss of viable myocardium or new regional wall motion abnormality, or (v) identification of an intracoronary thrombus by angiography or autopsy.

- Cardiac death with symptoms suggestive of myocardial ischemia and presumed new ischemic electrocardiographic changes or new left bundle branch block, but death occurred before cardiac biomarkers were obtained, or before cardiac biomarker values would be increased.

- Percutaneous coronary intervention related myocardial infarction is arbitrarily defined by elevation of cardiac troponin values $\left(>5 \times 99^{\text {th }}\right.$ percentile URL) in patients with normal baseline values $\left(\leq 99^{\text {th }}\right.$ percentile URL) or a rise of cardiac troponin values $>20 \%$ if the baseline values are elevated and are stable or falling. In addition, either (i) symptoms suggestive of myocardial ischemia, or (ii) new ischemic electrocardiographic changes, or (iii) angiographic findings consistent with a procedural complication, or (iv) imaging demonstration of new loss of viable myocardium or new regional wall motion abnormality are required.

- Stent thrombosis associated with myocardial infarction when detected by coronary angiography or autopsy in the setting of myocardial ischemia and with a rise and/or fall of cardiac biomarker values with at least one value above the $99^{\text {th }}$ percentile URL.

- Coronary artery bypass grafting related myocardial infarction is arbitrarily defined by elevation of cardiac biomarker values $\left(>10 \times 99^{\text {th }}\right.$ percentile URL) in patients with normal baseline cardiac troponin values $\left(\leq 99^{\text {th }}\right.$ percentile URL). In addition, either (i) new pathological Q waves or new left bundle branch block, or (ii) angiographic documented new graft or new native coronary artery occlusion, or (iii) imaging evidence of new loss of viable myocardium or new regional wall motion abnormality.

Criteria for prior myocardial infarction

Any one of the following criteria meets the diagnosis for prior myocardial infarction:

- Pathological Q waves with or without symptoms in the absence of nonischemic causes.

- Imaging evidence of a region of loss of viable myocardium that is thinned and fails to contract, in the absence of a nonischemic cause.

- Pathological findings of a prior myocardial infarction.

Abbreviation: URL, upper reference limit.

degradation products, increased cellular wall permeability, formation and release of membranous blebs, and myocyte necrosis. ${ }^{6}$ Regardless of the pathobiology, myocardial necrosis due to myocardial ischemia is designated as MI.

Also, histological evidence of myocardial injury with necrosis may be detectable in clinical conditions associated with predominantly nonischemic myocardial injury. Small amounts of myocardial injury with necrosis may be detected, which are associated with heart failure (HF), renal failure, myocarditis, arrhythmias, pulmonary embolism or otherwise uneventful percutaneous or surgical coronary procedures. These should not be labeled as MI or a complication of the procedures, but rather as myocardial injury, as illustrated in Figure 1. It is recognized that the complexity of clinical circumstances may sometimes render it difficult to determine where 


\section{REVIEWS}

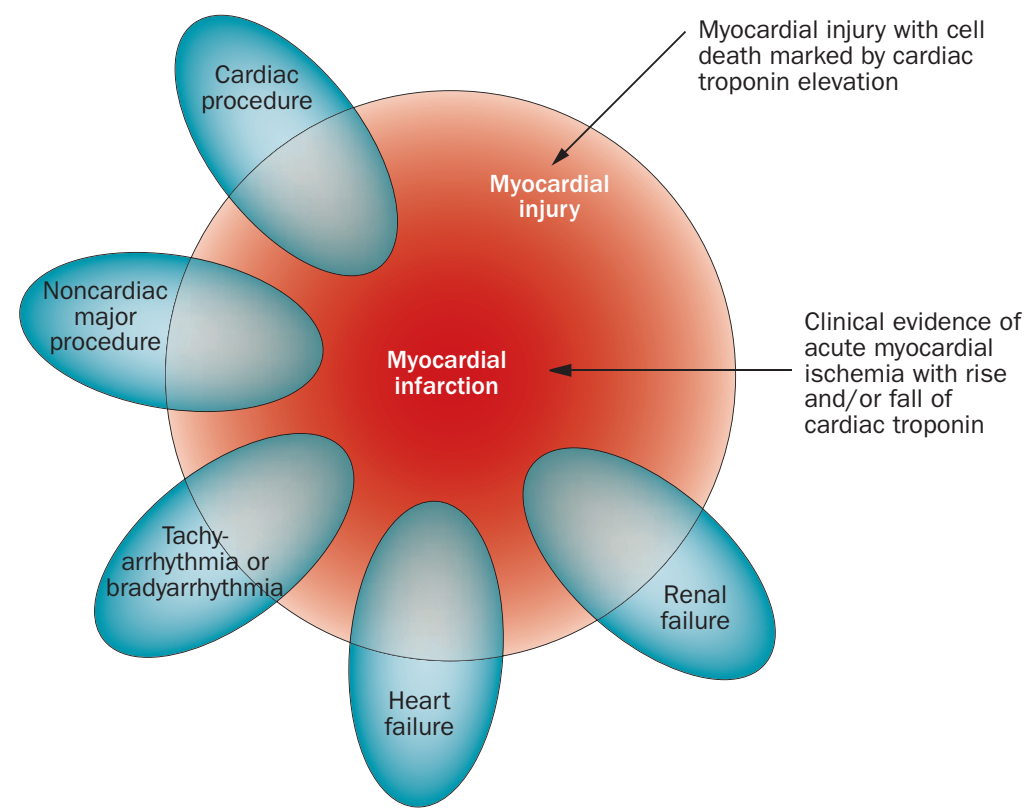

Figure 1 | This illustration shows various clinical entities—renal failure, heart failure, tachyarrhythmia or bradyarrhythmia, cardiac or noncardiac proceduresthat can be associated with myocardial injury with cell death marked by cardiac troponin elevation. However, these entities can also be associated with myocardial infarction in case of clinical evidence of acute myocardial ischemia with rise and/or fall of cardiac troponin.

individual cases may lie within the ovals of Figure 1. In this setting, it is important to distinguish acute causes of cTn elevation, which require a rise and/or fall of cTn values, from chronic elevations that tend not to change acutely. A list of such clinical circumstances associated with elevated values of cTn is presented in Table 1 . The multifactorial contributions resulting in the myocardial injury should be described in the patient record.

The preferred biomarker-overall and for each specific category of MI-is cTn (I or T), which has high myocardial tissue specificity as well as high clinical sensitivity. Detection of a rise and/or fall of the measurements is essential to the diagnosis of acute MI. ${ }^{7}$ An increased cTn concentration is defined as a value exceeding the $99^{\text {th }}$ percentile of a normal reference population (upper reference limit [URL]). This discriminatory $99^{\text {th }}$ percentile is designated as the decision level for the diagnosis of MI and must be determined for each specific assay with appropriate quality control in each laboratory. ${ }^{8,9}$ The values for the $99^{\text {th }}$ percentile URL defined by manufacturers, including those for many of the high-sensitivity assays in development, can be found in the package inserts for the assays or in recent publications. ${ }^{10-12}$

Values should be presented as nanograms per liter (ng/l) or picograms per milliliter $(\mathrm{pg} / \mathrm{ml})$ to make whole numbers. Criteria for the rise of cTn values are assaydependent, but can be defined from the precision profile of each individual assay, including high-sensitivity assays. ${ }^{10,11}$ Optimal precision, as described by coefficient of variation $(\mathrm{CV})$ at the $99^{\text {th }}$ percentile URL for each assay, should be defined as $\leq 10 \%$. Better precision (CV $\leq 10 \%$ ) allows for more sensitive assays and facilitates the detection of changing values. ${ }^{13}$ The use of assays that do not have optimal precision $\left(\mathrm{CV}>10 \%\right.$ at the $99^{\text {th }}$ percentile URL) makes determination of a significant change more difficult but does not cause false-positive results. Assays with CV $>20 \%$ at the $99^{\text {th }}$ percentile URL should not be used. ${ }^{13}$ It is acknowledged that preanalytic and analytic problems can induce elevated and reduced values of $\mathrm{cTn}^{10,11}$

Blood samples for the measurement of cTn should be drawn on first assessment and repeated 3-6h later. Later samples are required if further ischemic episodes occur, or when the timing of the initial symptoms is unclear. ${ }^{14}$ To establish the diagnosis of MI, a rise and/or fall in values with at least one value above the decision level is required, coupled with a strong pretest likelihood. The demonstration of a rising and/or falling pattern is needed to distinguish acute from chronic elevations in cTn concentrations that are associated with structural heart disease. ${ }^{10,11,15-19}$ For example, patients with renal failure or HF can have significant chronic elevations in cTn. These elevations can be marked, as seen in many patients with MI, but do not change acutely. ${ }^{7}$ However, a rising or falling pattern is not absolutely necessary to make the diagnosis of MI if a patient with a high pretest risk of MI presents late after symptom onset; for example, near the peak of the cTn time-concentration curve or on the slow-declining portion of that curve, when detecting a changing pattern can be problematic. Values may remain elevated for 2 weeks or more following the onset of myocyte necrosis. ${ }^{10}$

Sex-dependent values may be recommended for high-sensitivity troponin assays. ${ }^{20,21}$ An elevated cTn value ( $>99^{\text {th }}$ percentile URL), with or without a dynamic pattern of values or in the absence of clinical evidence of ischemia, should prompt a search for other diagnoses associated with myocardial injury, such as myocarditis, aortic dissection, pulmonary embolism, or HF. Renal failure and other more nonischemic chronic disease states, that can be associated with elevated cTn levels, are listed in Table $1 .^{10,11}$

If a cTn assay is not available, the best alternative is CKMB (measured by mass assay). As with troponin, an increased CKMB value is defined as a measurement above the $99^{\text {th }}$ percentile URL, which is designated as the decision level for the diagnosis of MI. ${ }^{22}$ Sex-specific values should be employed. ${ }^{22}$

\section{Clinical features of myocardial ischemia and infarction}

Onset of myocardial ischemia is the initial step in the development of MI and results from an imbalance between oxygen supply and demand. Myocardial ischemia in a clinical setting can usually be identified from the patient's history and from the ECG. Possible ischemic symptoms include various combinations of chest, upper extremity, mandibular or epigastric discomfort (with exertion or at rest) or an ischemic equivalent such as dyspnea or fatigue. The discomfort associated with acute MI usually lasts $>20 \mathrm{~min}$. Often, the discomfort is diffuse-not localized, nor positional, nor affected 
Table 1 | Elevations of cardiac troponin values because of myocardial injury

\begin{tabular}{|c|c|c|c|}
\hline $\begin{array}{l}\text { Injury related to primary } \\
\text { myocardial ischemia }\end{array}$ & $\begin{array}{l}\text { Injury related to supply/demand imbalance } \\
\text { of myocardial ischemia }\end{array}$ & $\begin{array}{l}\text { Injury not related to } \\
\text { myocardial ischemia }\end{array}$ & $\begin{array}{l}\text { Multifactorial or indeterminate } \\
\text { myocardial injury }\end{array}$ \\
\hline $\begin{array}{l}\text { Plaque rupture } \\
\text { Intraluminal coronary } \\
\text { artery thrombus formation }\end{array}$ & $\begin{array}{l}\text { Tachyarrhythmias or bradyarrhythmias } \\
\text { Aortic dissection or severe aortic valve disease } \\
\text { Hypertrophic cardiomyopathy } \\
\text { Cardiogenic, hypovolemic, or septic shock } \\
\text { Severe respiratory failure } \\
\text { Severe anemia } \\
\text { Hypertension with or without left ventricular hypertrophy } \\
\text { Coronary spasm } \\
\text { Coronary embolism or vasculitis } \\
\text { Coronary endothelial dysfunction without significant } \\
\text { coronary artery disease }\end{array}$ & $\begin{array}{l}\text { Cardiac contusion, surgery, } \\
\text { ablation, pacing, or } \\
\text { defibrillator shocks } \\
\text { Rhabdomyolysis with cardiac } \\
\text { involvement } \\
\text { Myocarditis } \\
\text { Cardiotoxic agents, } \\
\text { e.g. anthracyclines, herceptin }\end{array}$ & $\begin{array}{l}\text { Heart failure } \\
\text { Stress (Takotsubo) cardiomyopathy } \\
\text { Severe pulmonary embolism } \\
\text { or pulmonary hypertension } \\
\text { Sepsis and critically ill patients } \\
\text { Renal failure } \\
\text { Severe acute neurological diseases, } \\
\text { e.g. stroke, subarachnoid hemorrhage } \\
\text { Infiltrative diseases, e.g. amyloidosis, } \\
\text { sarcoidosis } \\
\text { Strenuous exercise }\end{array}$ \\
\hline
\end{tabular}

by movement of the region-and it may be accompanied by diaphoresis, nausea or syncope. However, these symptoms are not specific for myocardial ischemia. Accordingly, they may be misdiagnosed and attributed to gastrointestinal, neurological, pulmonary or musculoskeletal disorders. MI may occur with atypical symptoms-such as palpitations or cardiac arrest-or even without symptoms; for example in women, the elderly, diabetics, or postoperative and critically ill patients. ${ }^{2}$ Careful evaluation of these patients is advised, especially when there is a rising and/or falling pattern of cardiac biomarkers.

\section{Clinical classification of myocardial infarction}

For the sake of immediate treatment strategies, such as reperfusion therapy, it is usual practice to designate $\mathrm{MI}$ in patients with chest discomfort, or other ischemic symptoms that develop ST elevation in two contiguous leads (see ECG section), as an 'ST elevation MI' (STEMI). In contrast, patients without ST elevation at presentation are usually designated as having a 'non-ST elevation MI' (NSTEMI). Many patients with MI develop Q waves (Q wave MI), but others do not (non-Q MI). Patients without elevated biomarker values can be diagnosed as having unstable angina. In addition to these categories, MI is classified into various types, based on pathological, clinical and prognostic differences, along with different treatment strategies (Table 2).

\section{Spontaneous myocardial infarction (MI type 1)}

This is an event related to atherosclerotic plaque rupture, ulceration, fissuring, erosion, or dissection with resulting intraluminal thrombus in one or more of the coronary arteries, leading to decreased myocardial blood flow or distal platelet emboli with ensuing myocyte necrosis. The patient may have underlying severe CAD but, on occasion (5-20\%), nonobstructive or no CAD may be found at angiography, particularly in women. ${ }^{23-25}$

\section{Myocardial infarction secondary to an ischemic imbalance (MI type 2)}

In instances of myocardial injury with necrosis, where a condition other than CAD contributes to an imbalance between myocardial oxygen supply and/or demand, the term 'MI type 2' is employed (Figure 2). In critically ill patients, or in patients undergoing major (noncardiac)

Table 2 | Universal classification of myocardial infarction

\begin{tabular}{ll}
\hline Type & Description \\
\hline Type 1: & Spontaneous myocardial infarction related to atherosclerotic \\
spontaneous & plaque rupture, ulceration, fissuring, erosion, or dissection with \\
myocardial & resulting intraluminal thrombus in one or more of the coronary \\
infarction & arteries leading to decreased myocardial blood flow or distal \\
& platelet emboli with ensuing myocyte necrosis. The patient may \\
& have underlying severe coronary artery disease but on occasion \\
& nonobstructive or no coronary artery disease.
\end{tabular}

Type 2: myocardial infarction secondary to an ischemic imbalance

Type 3: myocardial infarction resulting in death when biomarker values are unavailable

Type 4a: myocardial infarction related to percutaneous coronary intervention

Type 4b: myocardia infarction related to stent thrombosis

Type 5: myocardial infarction related to coronary artery bypass grafting
In instances of myocardial injury with necrosis where a condition other than coronary artery disease contributes to an imbalance between myocardial oxygen supply and/or demand, e.g. coronary endothelial dysfunction, coronary artery spasm, coronary embolism, tachyarrhythmias or bradyarrhythmias, anemia, respiratory failure, hypotension, and hypertension with or without left ventricular hypertrophy.

Cardiac death with symptoms suggestive of myocardial ischemia and presumed new ischemic electrocardiographic changes or new left bundle branch block, but death occurring before blood samples could be obtained, before cardiac biomarkers could rise, or in rare cases cardiac biomarkers were not collected.

Myocardial infarction associated with percutaneous coronary intervention is arbitrarily defined by elevation of cardiac troponin values $>5 \times 99^{\text {th }}$ percentile URL in patients with normal baseline values ( $\leq 99^{\text {th }}$ percentile URL) or a rise of cardiac troponin values $>20 \%$ if the baseline values are elevated and are stable or falling. In addition, either (i) symptoms suggestive of myocardial ischemia, or (ii) new ischemic electrocardiographic changes or new left bundle branch block, or (iii) angiographic loss of patency of a major coronary artery or a side branch or persistent slow-flow or no-flow or embolization, or (iv) imaging demonstration of new loss of viable myocardium or new regional wall motion abnormality are required.

Myocardial infarction associated with stent thrombosis is detected by coronary angiography or autopsy in the setting of myocardial ischemia and with a rise and/or fall of cardiac biomarker values with at least one value above the $99^{\text {th }}$ percentile URL.

Myocardial infarction associated with coronary artery bypass grafting is arbitrarily defined by elevation of cardiac biomarker values $>10 \times 99^{\text {th }}$ percentile URL in patients with normal baseline cardiac troponin values $\left(\leq 99^{\text {th }}\right.$ percentile URL). In addition, either (i) new pathological $\mathrm{Q}$ waves or new left bundle branch block, or (ii) angiographic documented new graft or new native coronary artery occlusion, or (iii) imaging evidence of new loss of viable myocardium or new regional wall motion abnormality.

Abbreviation: URL, upper reference limit.

surgery, elevated values of cardiac biomarkers may appear, due to the direct toxic effects of endogenous or exogenous high circulating catecholamine levels. Also coronary vasospasm and/or endothelial dysfunction have the potential to cause MI. ${ }^{26-28}$ 

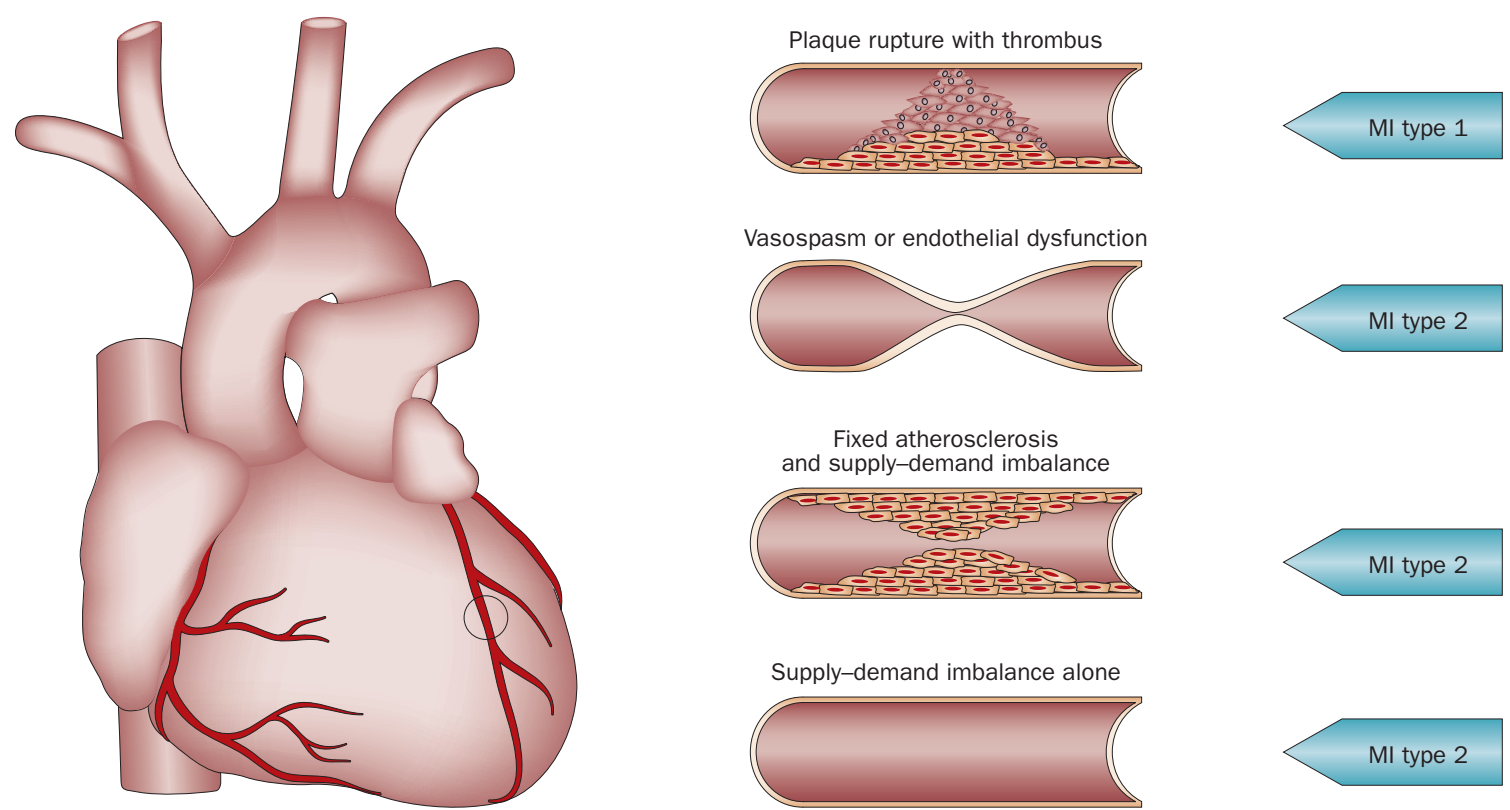

MI type 2

Fixed atherosclerosis and supply-demand imbalance

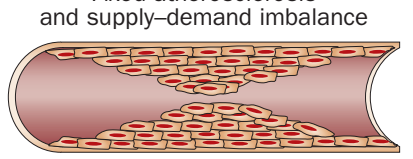

Supply-demand imbalance alone

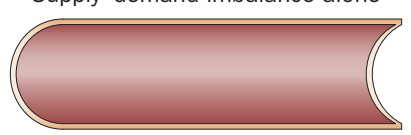

Figure 2 | Differentiation between MI types 1 and 2 according to the condition of the coronary arteries. Abbreviation: MI, myocardial infarction.

\section{Cardiac death due to myocardial infarction (MI type 3)}

Patients who suffer cardiac death, with symptoms suggestive of myocardial ischemia accompanied by presumed new ischemic ECG changes or new left bundle branch block (LBBB) - but without available biomarker values-represent a challenging diagnostic group. These individuals may die before blood samples for biomarkers can be obtained, or before elevated cardiac biomarkers can be identified. If patients present with clinical features of myocardial ischemia, or with presumed new ischemic ECG changes, they should be classified as having had a fatal MI, even if cardiac biomarker evidence of MI is lacking.

\section{Myocardial infarction associated with revascularization procedures (MI types 4 and 5)}

Periprocedural myocardial injury or infarction may occur at some stages in the instrumentation of the heart that is required during mechanical revascularization procedures, either by PCI or by coronary artery bypass grafting (CABG). Elevated cTn values may be detected following these procedures, since various insults may occur that can lead to myocardial injury with necrosis. ${ }^{29-32}$ It is likely that limitation of such injury is beneficial to the patient; however, a threshold for a worsening prognosis, related to an asymptomatic increase of cardiac biomarker values in the absence of procedural complications, is not well defined. ${ }^{33-35}$ Subcategories of PCI-related MI are connected to stent thrombosis and restenosis that may happen after the primary procedure.

\section{Electrocardiographic detection of myocardial infarction}

The ECG is an integral part of the diagnostic work-up of patients with suspected MI and should be acquired and interpreted promptly (i.e. target within $10 \mathrm{~min}$ ) after clinical presentation. ${ }^{2}$ Dynamic changes in the ECG waveforms during acute myocardial ischemic episodes often require acquisition of multiple ECGs, particularly if the ECG at initial presentation is nondiagnostic. Serial recordings in symptomatic patients with an initial nondiagnostic ECG should be performed at 15-30 min intervals or, if available, continuous computer-assisted 12-lead ECG recording. Recurrence of symptoms after an asymptomatic interval are an indication for a repeat tracing and, in patients with evolving ECG abnormalities, a predischarge ECG should be acquired as a baseline for future comparison. Acute or evolving changes in the ST-T waveforms and Q waves, when present, potentially allow the clinician to time the event, to identify the infarct-related artery, to estimate the amount of myocardium at risk as well as prognosis, and to determine therapeutic strategy. More profound ST-segment shift or $\mathrm{T}$ wave inversion involving multiple leads/territories is associated with a greater degree of myocardial ischemia and a worse prognosis. Other ECG signs associated with acute myocardial ischemia include cardiac arrhythmias, intraventricular and atrioventricular conduction delays, and loss of precordial $\mathrm{R}$ wave amplitude. Coronary artery size and distribution of arterial segments, collateral vessels, location, extent and severity of coronary stenosis, and prior myocardial necrosis can all impact ECG manifestations of myocardial ischemia. ${ }^{36}$ Therefore, the ECG at presentation should always be compared to prior ECG tracings, when available. The ECG by itself is often insufficient to diagnose acute myocardial ischemia or infarction, since ST deviation may be observed in other conditions, such as acute pericarditis, left ventricular hypertrophy (LVH), LBBB, Brugada syndrome, stress cardiomyopathy, and early repolarization patterns. ${ }^{37}$ Prolonged, new ST-segment elevation (e.g. >20 min), 
particularly when associated with reciprocal ST-segment depression, usually reflects acute coronary occlusion and results in myocardial injury with necrosis. As in cardiomyopathy, Q waves may also occur due to myocardial fibrosis in the absence of CAD.

ECG abnormalities of myocardial ischemia or infarction may be inscribed in the PR segment, the QRS complex, the ST segment or the T wave. The earliest manifestations of myocardial ischemia are typically $\mathrm{T}$ wave and ST-segment changes. Increased hyperacute T wave amplitude, with prominent symmetrical $\mathrm{T}$ waves in at least two contiguous leads, is an early sign that may precede the elevation of the ST segment. Transient Q waves may be observed during an episode of acute ischemia or (rarely) during acute MI with successful reperfusion. Table 3 lists ST-T wave criteria for the diagnosis of acute myocardial ischemia that may or may not lead to MI. The J point is used to determine the magnitude of the ST-segment shift. New, or presumed new, J-point elevation $\geq 0.1 \mathrm{mV}$ is required in all leads other than $\mathrm{V}_{2}$ and $\mathrm{V}_{3}$. In healthy men under age 40 , J point elevation can be as much as $0.25 \mathrm{mV}$ in leads $V_{2}$ or $V_{3}$, but it decreases with increasing age. Sex differences require different cut-points for women, since J point elevation in healthy women in leads $V_{2}$ and $V_{3}$ is less than in men. ${ }^{38}$ 'Contiguous leads' refers to lead groups such as anterior leads $\left(\mathrm{V}_{1}-\mathrm{V}_{6}\right)$, inferior leads (II, III, aVF) or lateral/apical leads (I, aVL). Supplemental leads such as $V_{3} R$ and $V_{4} R$ reflect the free wall of the right ventricle and $V_{7}-V_{9}$ the inferobasal wall.

The criteria in Table 3 require that the ST shift be present in two or more contiguous leads. For example, $\geq 0.2 \mathrm{mV}$ of ST elevation in lead $\mathrm{V}_{2}$, and $\geq 0.1 \mathrm{mV}$ in lead $\mathrm{V}_{1}$, would meet the criteria of two abnormal contiguous leads in a man $>40$ years old. However, $\geq 0.1 \mathrm{mV}$ and $<0.2 \mathrm{mV}$ of ST elevation, seen only in leads $\mathrm{V}_{2}-\mathrm{V}_{3}$ in men (or $<0.15 \mathrm{mV}$ in women), may represent a normal finding. It should be noted that, occasionally, acute myocardial ischemia may create sufficient ST-segment shift to meet the criteria in one lead, but have slightly less than the required ST shift in a contiguous lead. Lesser degrees of ST displacement or $\mathrm{T}$ wave inversion do not exclude acute myocardial ischemia or evolving MI, since a single static recording may miss the more dynamic ECG changes that might be detected with serial recordings. ST elevation or diagnostic Q waves in contiguous lead groups are more specific than ST depression in localizing the site of myocardial ischemia or necrosis..$^{39,40}$ Supplemental leads, as well as serial ECG recordings, should always be considered in patients that present with ischemic chest pain and a nondiagnostic initial ECG. ${ }^{41,42}$ ECG evidence of myocardial ischemia in the distribution of a left circumflex artery is often overlooked and is best captured using posterior leads at the fifth intercostal space $\left(\mathrm{V}_{7}\right.$ at the left posterior axillary line, $\mathrm{V}_{8}$ at the left midscapular line, and $\mathrm{V}_{9}$ at the left paraspinal border). Recording of these leads is strongly recommended in patients with high clinical suspicion for acute circumflex occlusion (for example, initial ECG nondiagnostic, or ST-segment depression in leads $\left.\mathrm{V}_{1}-\mathrm{V}_{3}\right){ }^{41}$ A cut-point of $0.05 \mathrm{mV}$ ST elevation is recommended in leads $\mathrm{V}_{7}-\mathrm{V}_{9}$; specificity is increased at a
Table 3 | Electrocardiographic manifestations of acute myocardial ischemia*

\begin{tabular}{|c|c|}
\hline Manifestation & Characteristics \\
\hline ST elevation & $\begin{array}{l}\text { New ST elevation at the J point in two contiguous leads with the } \\
\text { cut-points: } \geq 0.1 \mathrm{mV} \text { in all leads other than leads } V_{2}-V_{3} \text { where } \\
\text { the following cut-points apply: } \geq 0.2 \mathrm{mV} \text { in men } \geq 40 \text { years; } \geq 0.25 \mathrm{mV} \\
\text { in men }<40 \text { years, or } \geq 0.15 \mathrm{mV} \text { in women. }\end{array}$ \\
\hline $\begin{array}{l}\text { ST depression } \\
\text { and T wave } \\
\text { changes }\end{array}$ & $\begin{array}{l}\text { New horizontal or down-sloping ST depression } \geq 0.05 \mathrm{mV} \text { in two } \\
\text { contiguous leads and/or } T \text { inversion } \geq 0.1 \mathrm{mV} \text { in two contiguous } \\
\text { leads with prominent } \mathrm{R} \text { wave or } \mathrm{R} / \mathrm{S} \text { ratio }>1 \text {. }\end{array}$ \\
\hline
\end{tabular}

Table 4 | Electrocardiographic changes associated with prior myocardial infarction

\section{Characteristics}

Any $Q$ wave in leads $V_{2}-V_{3} \geq 0.02 s$ or $Q S$ complex in leads $V_{2}$ and $V_{3}$.

$\mathrm{Q}$ wave $\geq 0.03 \mathrm{~s}$ and $\geq 0.1 \mathrm{mV}$ deep or QS complex in leads I, II, aVL, aVF, or $\mathrm{V}_{4}-\mathrm{V}_{6}$ in any two leads of a contiguous lead grouping (I, aVL; $\mathrm{V}_{1}-\mathrm{V}_{6}$; II, III, aVF).*

$R$ wave $\geq 0.04 \mathrm{~s}$ in $\mathrm{V}_{1}-\mathrm{V}_{2}$ and $\mathrm{R} / \mathrm{S} \geq 1$ with a concordant positive $T$ wave in absence of conduction defect.

*The same criteria are used for supplemental leads $\mathrm{V}_{7}-\mathrm{V}_{9}$.

cut point $\geq 0.1 \mathrm{mV}$ ST elevation and this cut-point should be used in men $<40$ years old. ST depression in leads $\mathrm{V}_{1}-\mathrm{V}_{3}$ may be suggestive of inferobasal myocardial ischemia (posterior infarction), especially when the terminal $\mathrm{T}$ wave is positive (ST elevation equivalent), however this is nonspecific. ${ }^{41-43}$ In patients with inferior and suspected right ventricular infarction, right precordial leads $\mathrm{V}_{3} \mathrm{R}$ and $\mathrm{V}_{4} \mathrm{R}$ should be recorded, since $\mathrm{ST}$ elevation $\geq 0.05 \mathrm{mV}$ ( $\geq 0.1 \mathrm{mV}$ in men $<30$ years old) provides supportive criteria for the diagnosis. ${ }^{42}$

During an episode of acute chest discomfort, pseudonormalization of previously inverted $\mathrm{T}$ waves may indicate acute myocardial ischemia. Pulmonary embolism, intracranial processes, electrolyte abnormalities, hypothermia, pericarditis or myocarditis may also result in ST-T abnormalities and should be considered in the differential diagnosis. The diagnosis of MI is more difficult in the presence of LBBB. ${ }^{44,45}$ However, concordant ST-segment elevation or a previous ECG may be helpful to determine the presence of acute $\mathrm{MI}$ in this setting. In patients with right bundle branch block (RBBB), ST-T abnormalities in leads $\mathrm{V}_{1}-\mathrm{V}_{3}$ are common, making it difficult to assess the presence of ischemia in these leads; however, when new ST elevation or Q waves are found, myocardial ischemia or infarction should be considered.

\section{Prior myocardial infarction}

As shown in Table 4, Q waves or QS complexes in the absence of QRS confounders are pathognomonic of a prior MI in patients with ischemic heart disease, regardless of symptoms. ${ }^{46,47}$ The specificity of the ECG diagnosis for MI is greatest when Q waves occur in several leads or lead groupings. When the $\mathrm{Q}$ waves are associated with ST deviations or T wave changes in the same leads, the likelihood of MI is increased; for example, minor Q waves $\geq 0.02 \mathrm{~s}$ and $<0.03 \mathrm{~s}$ that are $\geq 0.1 \mathrm{mV}$ deep are suggestive of prior MI if accompanied by inverted $\mathrm{T}$ waves in the same lead group. Other validated MI coding 
Table 5 | Common electrocardiographic pitfalls in diagnosing myocardial infarction

\begin{tabular}{|c|c|}
\hline False positives & False negatives \\
\hline $\begin{array}{l}\text { Early repolarization } \\
\text { Left bundle branch block } \\
\text { Pre-excitation } \\
\text { J point elevation syndromes, e.g. Brugada syndrome } \\
\text { Pericarditis or myocarditis } \\
\text { Pulmonary embolism } \\
\text { Subarachnoid hemorrhage } \\
\text { Metabolic disturbances such as hyperkalemia } \\
\text { Cardiomyopathy } \\
\text { Lead transposition } \\
\text { Cholecystitis } \\
\text { Persistent juvenile pattern } \\
\text { Malposition of precordial electrocardiogram electrodes } \\
\text { Tricyclic antidepressants or phenothiazines }\end{array}$ & $\begin{array}{l}\text { Prior myocardial infarction } \\
\text { with Q waves and/or persistent } \\
\text { ST elevation } \\
\text { Right ventricular pacing } \\
\text { Left bundle branch block }\end{array}$ \\
\hline
\end{tabular}

algorithms, such as the Minnesota Code and WHO multinational MONitoring of trends and determinants in CArdiovascular disease (MONICA), have been used in epidemiological studies and clinical trials. ${ }^{3}$

\section{Silent myocardial infarction}

Asymptomatic patients who develop new pathological Q wave criteria for MI detected during routine ECG follow-up, or reveal evidence of MI by cardiac imaging, that cannot be directly attributed to a coronary revascularization procedure, should be termed 'silent MI'. ${ }^{48-51}$ In studies, silent Q wave MI accounted for 9-37\% of all nonfatal MI events and were associated with a significantly increased mortality risk. ${ }^{48,49}$ Improper lead placement or QRS confounders may result in what appear to be new Q waves or QS complexes, as compared to a prior tracing. Thus, the diagnosis of a new silent $\mathrm{Q}$ wave MI should be confirmed by a repeat ECG with correct lead placement, or by an imaging study, and by focused questioning about potential interim ischemic symptoms.

\section{Conditions that confound the ECG diagnosis of myocardial infarction}

A QS complex in lead $V_{1}$ is normal. A Q wave $<0.03$ s and $<25 \%$ of the $\mathrm{R}$ wave amplitude in lead III is normal if the frontal QRS axis is between $-30^{\circ}$ and $0^{\circ}$. A Q wave may also be normal in aVL if the frontal QRS axis is between $60^{\circ}$ and $90^{\circ}$. Septal Q waves are small, nonpathological Q waves $<0.03 \mathrm{~s}$ and $<25 \%$ of the R-wave amplitude in leads I, aVL, aVF, and $\mathrm{V}_{4}-\mathrm{V}_{6}$. Pre-excitation, obstructive, dilated or stress cardiomyopathy, cardiac amyloidosis, LBBB, left anterior hemiblock, LVH, right ventricular hypertrophy, myocarditis, acute cor pulmonale, or hyperkalemia may be associated with Q waves or QS complexes in the absence of MI. ECG abnormalities that mimic myocardial ischemia or MI are presented in Table 5.

\section{Imaging techniques}

Noninvasive imaging plays many roles in patients with known or suspected MI, but this section concerns only its role in the diagnosis and characterization of MI. The underlying rationale is that regional myocardial hypoperfusion and ischemia lead to a cascade of events, including myocardial dysfunction, cell death and healing by fibrosis. Important imaging parameters are therefore perfusion, myocyte viability, myocardial thickness, thickening and motion, and the effects of fibrosis on the kinetics of paramagnetic or radio-opaque contrast agents.

Commonly used imaging techniques in acute and chronic infarction are echocardiography, radionuclide ventriculography, myocardial perfusion scintigraphy (MPS) using single photon emission computed tomography (SPECT), and magnetic resonance imaging (MRI). Positron emission tomography (PET) and X-ray computed tomography (CT) are less common. ${ }^{52}$ There is considerable overlap in their capabilities and each of the techniques can, to a greater or lesser extent, assess myocardial viability, perfusion, and function. Only the radionuclide techniques provide a direct assessment of myocyte viability, because of the inherent properties of the tracers used. Other techniques provide indirect assessments of myocardial viability, such as contractile response to dobutamine by echocardiography or myocardial fibrosis by MR.

\section{Echocardiography}

The strength of echocardiography is the assessment of cardiac structure and function, in particular myocardial thickness, thickening and motion. Echocardiographic contrast agents can improve visualization of the endocardial border and can be used to assess myocardial perfusion and microvascular obstruction. Tissue Doppler and strain imaging permit quantification of global and regional function. ${ }^{53}$ Intravascular echocardiographic contrast agents have been developed that target specific molecular processes, but these techniques have not yet been applied in the setting of MI. ${ }^{54}$

\section{Radionuclide imaging}

Several radionuclide tracers allow viable myocytes to be imaged directly, including the SPECT tracers thallium201, technetium-99m MIBI and tetrofosmin, and the PET tracers F-2-fluorodeoxyglucose (FDG) and rubidium82. ${ }^{18,52}$ The strength of the SPECT techniques is that these are the only commonly available direct methods of assessing viability, although the relatively low resolution of the images leaves them at a disadvantage for detecting small areas of MI. The common SPECT radiopharmaceuticals are also tracers of myocardial perfusion and the techniques thereby readily detect areas of MI and inducible perfusion abnormalities. ECG-gated imaging provides a reliable assessment of myocardial motion, thickening and global function. Evolving radionuclide techniques that are relevant to the assessment of MI include imaging of sympathetic innervation using iodine-123-labeled meta-iodo-benzylguanidine (mIBG), ${ }^{55}$ imaging of matrix metalloproteinase activation in ventricular remodeling, ${ }^{56,57}$ and refined assessment of myocardial metabolism. ${ }^{58}$

\section{Magnetic resonance imaging}

The high tissue contrast of cardiovascular MRI provides an accurate assessment of myocardial function and it has similar capability to echocardiography in suspected acute MI. Paramagnetic contrast agents can be used to assess 
myocardial perfusion and the increase in extracellular space that is associated with the fibrosis of prior MI. These techniques have been used in the setting of acute $\mathrm{MI},{ }^{59,60}$ and imaging of myocardial fibrosis by delayed contrast enhancement is able to detect even small areas of subendocardial MI. It is also of value in detecting myocardial disease states that can mimic MI, such as myocarditis. ${ }^{61}$

\section{Computed tomography}

Infarcted myocardium is initially visible as a focal area of decreased left ventricle (LV) enhancement, but later imaging shows hyperenhancement, as with late gadolinium imaging by MRI. ${ }^{62}$ This finding is clinically relevant because contrast-enhanced CT may be performed for suspected pulmonary embolism and aortic dissectionconditions with clinical features that overlap with those of acute MI-but the technique is not used routinely. Similarly, CT assessment of myocardial perfusion is technically feasible but not yet fully validated.

\section{Applying imaging in acute myocardial infarction}

Imaging techniques can be useful in the diagnosis of acute MI because of their ability to detect wall motion abnormalities or loss of viable myocardium in the presence of elevated cardiac biomarker values. If, for some reason, biomarkers have not been measured or may have normalized, demonstration of new loss of myocardial viability in the absence of nonischemic causes meets the criteria for MI. Normal function and viability have a very high negative predictive value and practically exclude acute MI. ${ }^{63}$ Thus, imaging techniques are useful for early triage and discharge of patients with suspected MI. However, if biomarkers have been measured at appropriate times and are normal, this excludes an acute MI and takes precedence over the imaging criteria.

Abnormal regional myocardial motion and thickening may be caused by acute MI or by one or more of several other conditions, including prior MI, acute ischemia, stunning or hibernation. Nonischemic conditions, such as cardiomyopathy and inflammatory or infiltrative diseases, can also lead to regional loss of viable myocardium or functional abnormality. Therefore, the positive predictive value of imaging for acute MI is not high unless these conditions can be excluded, and unless a new abnormality is detected or can be presumed to have arisen in the setting of other features of acute MI.

Echocardiography provides an assessment of many nonischemic causes of acute chest pain, such as perimyocarditis, valvular heart disease, cardiomyopathy, pulmonary embolism or aortic dissection. ${ }^{53}$ It is the imaging technique of choice for detecting complications of acute MI, including myocardial free wall rupture, acute ventricular septal defect, and mitral regurgitation secondary to papillary muscle rupture or ischemia.

Radionuclide imaging can be used to assess the amount of myocardium that is salvaged by acute revascularization. ${ }^{64}$ Tracer is injected at the time of presentation, with imaging deferred until after revascularization, providing a measure of myocardium at risk. Before discharge, a second resting injection provides a measure of final infarct size, and the difference between the two corresponds to the myocardium that has been salvaged.

\section{Applying imaging in late presentation of myocardial infarction}

In case of late presentation after suspected MI, the presence of regional wall motion abnormality, thinning or scar in the absence of nonischemic causes, provides evidence of past MI. The high resolution and specificity of late gadolinium enhancement MRI for the detection of myocardial fibrosis has made this a very valuable technique. In particular, the ability to distinguish between subendocardial and other patterns of fibrosis provides a differentiation between ischemic heart disease and other myocardial abnormalities. Imaging techniques are also useful for risk stratification after a definitive diagnosis of MI. The detection of residual or remote ischemia and/or ventricular dysfunction provides powerful indicators of later outcome.

\section{Diagnostic criteria for myocardial infarction with PCI (MI type 4)}

Balloon inflation during PCI often causes transient ischemia, whether or not it is accompanied by chest pain or ST-T changes. Myocardial injury with necrosis may result from recognizable periprocedural events-alone or in combination-such as coronary dissection, occlusion of a major coronary artery or a side-branch, disruption of collateral flow, slow flow or no-reflow, distal embolization, and microvascular plugging. Embolization of intracoronary thrombus or atherosclerotic particulate debris may not be preventable, despite current anticoagulant and antiplatelet adjunctive therapy, aspiration or protection devices. Such events induce inflammation of the myocardium surrounding islets of myocardial necrosis. ${ }^{65} \mathrm{New}$ areas of myocardial necrosis have been demonstrated by MRI following PCI. ${ }^{6}$

The occurrence of procedure-related myocardial cell injury with necrosis can be detected by measurement of cardiac biomarkers before the procedure, repeated 3-6 h later and, optionally, further remeasurement $12 \mathrm{~h}$ thereafter. Increasing levels can only be interpreted as procedure-related myocardial injury if the preprocedural cTn value is normal ( $\leq 99^{\text {th }}$ percentile URL) or if levels are stable or falling. ${ }^{67,68}$ In patients with normal preprocedural values, elevation of cardiac biomarker values above the $99^{\text {th }}$ percentile URL following PCI are indicative of procedure-related myocardial injury. In earlier studies, increased values of postprocedural cardiac biomarkers, especially CKMB, were associated with impaired outcome. ${ }^{69,70}$ However, when cTn concentrations are normal before PCI and become abnormal after the procedure, the threshold above the $99^{\text {th }}$ percentile URL-whereby an adverse prognosis is evident-is not well defined ${ }^{71}$ and it is debatable whether such a threshold even exists. ${ }^{72}$ If a single baseline cTn value is elevated, it is impossible to determine whether further increases are due to the procedure or to the initial process causing the elevation. In this situation, it appears that the prognosis is largely determined by the preprocedural cTn level. ${ }^{71}$ 
These relationships will probably become even more complex for the new high-sensitivity troponin assays. ${ }^{70}$

In patients undergoing $\mathrm{PCI}$ with normal $\left(\leq 99^{\text {th }}\right.$ percentile URL) baseline cTn concentrations, elevations of cTn $>5 \times 99^{\text {th }}$ percentile URL occurring within $48 \mathrm{~h}$ of the procedure-plus either (i) evidence of prolonged ischemia ( $\geq 20 \mathrm{~min}$ ) as demonstrated by prolonged chest pain, or (ii) ischemic ST changes or new pathological Q waves, or (iii) angiographic evidence of a flow limiting complication, such as of loss of patency of a side branch, persistent slow-flow or no-reflow, embolization, or (iv) imaging evidence of new loss of viable myocardium or new regional wall motion abnormality-is defined as PCI-related MI (type 4a). This threshold of cTn values $>5 \times 99^{\text {th }}$ percentile URL is arbitrarily chosen, based on clinical judgment and societal implications of the label of periprocedural MI. When a cTn value is $\leq 5 \times 99^{\text {th }}$ percentile URL after PCI and the $\mathrm{cTn}$ value was normal before the PCI-or when the $c T n$ value is $>5 \times 99^{\text {th }}$ percentile URL in the absence of ischemic, angiographic or imaging findings-the term 'myocardial injury' should be used.

If the baseline cTn values are elevated and are stable or falling, then a rise of $>20 \%$ is required for the diagnosis of a type 4a MI, as with reinfarction. Recent data suggest that, when PCI is delayed after MI until biomarker concentrations are falling or have normalized, and elevation of cardiac biomarker values then reoccurs, this may have some long-term significance. However, additional data are needed to confirm this finding. ${ }^{73}$

A subcategory of PCI-related MI is stent thrombosis, as documented by angiography and/or at autopsy and a rise and/or fall of cTn values $>99^{\text {th }}$ percentile URL (identified as MI type $4 \mathrm{~b}$ ). In order to stratify the occurrence of stent thrombosis in relation to the timing of the PCI procedure, the Academic Research Consortium recommends temporal categories of 'early' ( 0 to 30 days), 'late' (31 days to 1 year), and 'very late' ( $>1$ year) to distinguish likely differences in the contribution of the various pathophysiological processes during each of these intervals. ${ }^{74}$ Occasionally, MI occurs in the clinical setting of what appears to be a stent thrombosis; however, at angiography, restenosis is observed without evidence of thrombus (see section on clinical trials).

\section{Diagnostic criteria for myocardial infarction with CABG (MI type 5)}

During CABG, numerous factors can lead to periprocedural myocardial injury with necrosis. These include direct myocardial trauma from (i) suture placement or manipulation of the heart, (ii) coronary dissection, (iii) global or regional ischemia related to inadequate intraoperative cardiac protection, (iv) microvascular events related to reperfusion, (v) myocardial injury induced by oxygen free radical generation, or (vi) failure to reperfuse areas of the myocardium that are not subtended by graftable vessels. ${ }^{75-77}$ MRI studies suggest that most necrosis in this setting is not focal but diffuse and localized in the subendocardium. ${ }^{78}$

In patients with normal values before surgery, any increase of cardiac biomarker values after $C A B G$ indicates myocardial necrosis, implying that an increasing magnitude of biomarker concentrations is likely to be related to an impaired outcome. This has been demonstrated in clinical studies employing CKMB, where elevations 5, 10 and 20 times the URL after CABG were associated with worsened prognosis; similarly, impaired outcome has been reported when cTn values were elevated to the highest quartile or quintile of the measurements. ${ }^{79-83}$

Unlike prognosis, scant literature exists concerning the use of biomarkers for defining an MI related to a primary vascular event in a graft or native vessel in the setting of CABG. In addition, when the baseline cTn value is elevated $\left(>99^{\text {th }}\right.$ percentile URL), higher levels of biomarker values are seen post-CABG. Therefore, biomarkers cannot stand alone in diagnosing $\mathrm{MI}$ in this setting. In view of the adverse impact on survival observed in patients with significant elevation of biomarker concentrations, this Task Force suggests, by arbitrary convention, that cTn values $>10 \times 99^{\text {th }}$ percentile URL during the first $48 \mathrm{~h}$ following $\mathrm{CABG}$, occurring from a normal baseline $\mathrm{cTn}$ value $\left(\leq 99^{\text {th }}\right.$ percentile URL). In addition, either (i) new pathological Q waves or new LBBB, or (ii) angiographically documented new graft or new native coronary artery occlusion, or (iii) imaging evidence of new loss of viable myocardium or new regional wall motion abnormality, should be considered as diagnostic of a CABG-related MI (type 5). Cardiac biomarker release is considerably higher after valve replacement with CABG than with bypass surgery alone, and with on-pump CABG compared to off-pump CABG ${ }^{84}$ The threshold described above is more robust for isolated on-pump CABG. As for $\mathrm{PCI}$, the existing principles from the universal definition of MI should be applied for the definition of MI $>48 \mathrm{~h}$ after surgery.

\section{Assessment of MI in patients undergoing other cardiac procedures}

New ST-T abnormalities are common in patients who undergo cardiac surgery. When new pathological $\mathrm{Q}$ waves appear in different territories than those identified before surgery, MI (types 1 or 2) should be considered, particularly if associated with elevated cardiac biomarker values, new wall motion abnormalities or hemodynamic instability.

Novel procedures such as transcatheter aortic valve implantation (TAVI) or mitral clip may cause myocardial injury with necrosis, both by direct trauma to the myocardium and by creating regional ischemia from coronary obstruction or embolization. It is likely that, similarly to $\mathrm{CABG}$, the more marked the elevation of the biomarker values, the worse the prognosis-but data on that are not available.

Modified criteria have been proposed for the diagnosis of periprocedural $\mathrm{MI} \leq 72 \mathrm{~h}$ after aortic valve implantation. ${ }^{85}$ However, given that there is too little evidence, it appears reasonable to apply the same criteria for procedure-related MI as stated above for CABG.

Ablation of arrhythmias involves controlled myocardial injury with necrosis, by application of warming or cooling of the tissue. The extent of the injury with necrosis can 
be assessed by cTn measurement; however, an elevation of cTn values in this context should not be labeled as MI.

\section{Myocardial infarction associated with noncardiac procedures}

Perioperative $\mathrm{MI}$ is the most common major perioperative vascular complication in major noncardiac surgery, and is associated with a poor prognosis. ${ }^{86,87}$ Most patients who have a perioperative MI will not experience ischemic symptoms. Nevertheless, asymptomatic perioperative MI is as strongly associated with 30-day mortality, as is symptomatic MI ${ }^{86}$ Routine monitoring of cardiac biomarkers in high-risk patients, both prior to and $48-72 \mathrm{~h}$ after major surgery, is therefore recommended. Measurement of high-sensitivity cTn in postoperative samples reveals that $45 \%$ of patients have levels above the $99^{\text {th }}$ percentile URL and $22 \%$ have an elevation and a rising pattern of values indicative of evolving myocardial necrosis ${ }^{88}$ Studies of patients undergoing major noncardiac surgery strongly support the idea that many of the infarctions diagnosed in this context are caused by a prolonged imbalance between myocardial oxygen supply and demand, against a background of CAD ${ }^{89,90}$ Together with a rise and/or fall of cTn values, this indicates MI type 2. However, one pathological study of fatal perioperative MI patients showed plaque rupture and platelet aggregation, leading to thrombus formation, in approximately half of such events; ${ }^{91}$ that is to say, MI type 1. Given the differences that probably exist in the therapeutic approaches to each, close clinical scrutiny and judgment are needed.

\section{Myocardial infarction in the intensive care unit} Elevations of cTn values are common in patients in the intensive care unit and are associated with adverse prognosis, regardless of the underlying disease state. ${ }^{92,93}$ Some elevations may reflect MI type 2 due to underlying $\mathrm{CAD}$ and increased myocardial oxygen demand..$^{94}$ Other patients may have elevated values of cardiac biomarkers, due to myocardial injury with necrosis induced by catecholamine or direct toxic effect from circulating toxins. Moreover, in some patients, MI type 1 may occur. It is often a challenge for the clinician, caring for a critically ill patient with severe single organ or multiorgan pathology, to decide on a plan of action when the patient has elevated cTn values. If and when the patient recovers from the critical illness, clinical judgment should be employed to decide whether-and to what extent-further evaluation for CAD or structural heart disease is indicated. ${ }^{95}$

\section{Recurrent myocardial infarction}

'Incident MI' is defined as the individual's first MI. When features of MI occur in the first 28 days after an incident event, this is not counted as a new event for epidemiological purposes. If characteristics of MI occur after 28 days following an incident MI, it is considered to be a recurrent MI. ${ }^{3}$

\section{Reinfarction}

The term 'reinfarction' is used for an acute MI that occurs within 28 days of an incident or recurrent MI. ${ }^{3}$ The ECG diagnosis of suspected reinfarction following the initial MI may be confounded by the initial evolutionary ECG changes. Reinfarction should be considered when ST elevation $\geq 0.1 \mathrm{mV}$ recurs, or new pathognomonic $\mathrm{Q}$ waves appear, in at least two contiguous leads, particularly when associated with ischemic symptoms for $20 \mathrm{~min}$ or longer. Re-elevation of the ST-segment can, however, also be seen in threatened myocardial rupture and should lead to additional diagnostic workup. ST depression or LBBB alone are nonspecific findings and should not be used to diagnose reinfarction.

In patients in whom reinfarction is suspected from clinical signs or symptoms following the initial MI, an immediate measurement of $\mathrm{cTn}$ is recommended. A second sample should be obtained 3-6h later. If the cTn concentration is elevated, but stable or decreasing at the time of suspected reinfarction, the diagnosis of reinfarction requires a $20 \%$ or greater increase of the cTn value in the second sample. If the initial cTn concentration is normal, the criteria for new acute MI apply.

\section{Myocardial injury or infarction associated with heart failure}

Depending on the assay used, detectable-to-clearly elevated cTn values, indicative of myocardial injury with necrosis, may be seen in patients with HF syndrome. ${ }^{96}$ Using high-sensitivity cTn assays, measurable cTn concentrations may be present in nearly all patients with HF, with a significant percentage exceeding the $99^{\text {th }}$ percentile URL, particularly in those with more severe HF syndrome, such as in acutely decompensated $\mathrm{HF}^{97}$

While MI type 1 is an important cause of acutely decompensated HF-and should always be considered in the context of an acute presentation-elevated cTn values alone, in a patient with HF syndrome, do not establish the diagnosis of MI type 1 and may, indeed, be seen in those with nonischemic HF. Beyond MI type 1, multiple mechanisms have been invoked to explain measurable-topathologically elevated cTn concentrations in patients with HF. ${ }^{66,97}$ For example, MI type 2 may result from increased transmural pressure, small-vessel coronary obstruction, endothelial dysfunction, anemia or hypotension. Besides MI type 1 or 2, cardiomyocyte apoptosis and autophagy due to wall stretch has been experimentally demonstrated. Direct cellular toxicity related to inflammation, circulating neurohormones, infiltrative processes, as well as myocarditis and stress cardiomyopathy, may present with HF and abnormal cTn measurement. ${ }^{97}$

Whilst prevalent and complicating the diagnosis of MI, the presence, magnitude and persistence of cTn elevation in HF is increasingly accepted to be an independent predictor of adverse outcomes in both acute and chronic HF syndrome, irrespective of mechanism, and should not be discarded as 'false positive.97,98

In the context of an acutely decompensated HF presentation, cTn I or T should always be promptly measured and ECG recorded, with the goal of identifying or excluding MI type 1 as the precipitant. In this setting, elevated cTn values should be interpreted with a high level of suspicion for MI type 1 if a significant rise and/or fall 


\section{REVIEWS}

Table 6 | Tabulation in clinical trials of MI types according to multiples of the $99^{\text {th }}$ percentile URL of the applied cardiac biomarker

\begin{tabular}{|c|c|c|c|c|c|c|c|}
\hline Multiples $\times 99 \%$ & $\begin{array}{l}\text { MI type 1: } \\
\text { spontaneous }\end{array}$ & $\begin{array}{l}\text { MI type 2: } \\
\text { secondary }\end{array}$ & $\begin{array}{l}\text { MI type 3: } \\
\text { death* }\end{array}$ & $\begin{array}{l}\text { MI type 4a: } \\
\text { PCI }\end{array}$ & $\begin{array}{l}\text { MI type } 4 \mathrm{~b}: \\
\text { stent thrombosis }\end{array}$ & $\begin{array}{l}\text { MI type 4c: } \\
\text { restenosis }{ }^{\ddagger}\end{array}$ & $\begin{array}{l}\text { MI type 5: } \\
\text { CABG }\end{array}$ \\
\hline \multicolumn{8}{|l|}{$1-3$} \\
\hline \multicolumn{8}{|l|}{$3-5$} \\
\hline \multicolumn{8}{|l|}{$5-10$} \\
\hline \multicolumn{8}{|l|}{$>10$} \\
\hline
\end{tabular}

*Biomarker values are unavailable because of death before blood samples are obtained (blue area). Red areas indicate arbitrarily defined cardiac troponin values below the MI decision limit whether PCl or CABG. FRestenosis is defined as $\geq 50 \%$ stenosis at coronary angiography or a complex lesion associated with a rise and/or fall of cardiac troponin values $>99^{\text {th }}$ percentile URL

and no other significant obstructive coronary artery disease of greater severity following: (i) initially successful stent deployment, or (ii) dilatation of a coronary artery stenosis with balloon

angioplasty $(<50 \%)$. Abbreviations: CABG, coronary artery bypass grafting; MI, myocardial infarction; PCI, percutaneous coronary intervention; URL, upper reference limit.

of the marker are seen, or if it is accompanied by ischemic symptoms, new ischemic ECG changes or loss of myocardial function on noninvasive testing. Coronary artery anatomy may often be well-known; such knowledge may be used to interpret abnormal troponin results. If normal coronary arteries are present, either a type $2 \mathrm{MI}$ or a noncoronary mechanism for troponin release may be invoked..$^{97}$

On the other hand, when coronary anatomy is not established, the recognition of a cTn value in excess of the $99^{\text {th }}$ percentile URL alone is not sufficient to make a diagnosis of acute $\mathrm{MI}$ due to $\mathrm{CAD}$, nor is it able to identify the mechanism for the abnormal cTn value. In this setting, further information, such as myocardial perfusion studies, coronary angiography, or MRI is often required to better understand the cause of the abnormal cTn measurement. However, it may be difficult to establish the reason for cTn abnormalities, even after such investigations..$^{96,97}$

\section{Application of MI in clinical trials and quality assurance programs}

In clinical trials, MI may be an entry criterion or an end point. A universal definition for MI is of great benefit for clinical studies, since it will allow a standardized approach for interpretation and comparison across different trials. The definition of MI as an entry criterion, e.g. MI type 1 and not MI type 2, will determine patient characteristics in the trial. Occasionally MI occurs and, at angiography, restenosis is the only angiographic explanation. ${ }^{99,100}$ This PCI-related MI type might be designated as an 'MI type 4 c', defined as $\geq 50 \%$ stenosis at coronary angiography or a complex lesion associated with a rise and/or fall of cTn values $>99^{\text {th }}$ percentile URL and no other significant obstructive CAD of greater severity following: (i) initially successful stent deployment or (ii) dilatation of a coronary artery stenosis with balloon angioplasty $(<50 \%)$.

In recent investigations, different MI definitions have been employed as trial outcomes, thereby hampering comparison and generalization between these trials. Consistency among investigators and regulatory authorities, with regard to the definition of MI used as an end point in clinical investigations, is of substantial value. Adaptation of the definition to an individual clinical study may be appropriate in some circumstances and should have a well-articulated rationale. No matter what, investigators should ensure that a trial provides comprehensive data for the various types of MI and includes the $99^{\text {th }}$ percentile URL decision limits of cTn or other biomarkers employed. Multiples of the $99^{\text {th }}$ percentiles URL may be indicated as shown in Table 6 . This will facilitate comparison of trials and meta-analyses.

Because different assays may be used, including newer, higher-sensitivity cTn assays in large multicenter clinical trials, it is advisable to consistently apply the $99^{\text {th }}$ percentile URL. This will not totally harmonize troponin values across different assays, but will improve the consistency of the results. In patients undergoing cardiac procedures, the incidence of MI may be used as a measure of quality, provided that a consistent definition is applied by all centers participating in the quality assurance program. To be effective and to avoid bias, this type of assessment will need to develop a paradigm to harmonize the different cTn assay results across sites.

\section{Public policy implications of the adjustment of the MI definition}

Revision of the definition of MI has a number of implications for individuals as well as for society at large. A tentative or final diagnosis is the basis for advice about further diagnostic testing, lifestyle changes, treatment and prognosis for the patient. The aggregate of patients with a particular diagnosis is the basis for health care planning and policy and resource allocation.

One of the goals of good clinical practice is to reach a definitive and specific diagnosis, which is supported by current scientific knowledge. The approach to the definition of MI outlined in this document meets this goal. In general, the conceptual meaning of the term 'myocardial infarction' has not changed, although new, sensitive diagnostic methods have been developed to diagnose this entity. Thus, the diagnosis of acute MI is a clinical diagnosis based on patient symptoms, ECG changes, and highly sensitive biochemical markers, as well as information gleaned from various imaging techniques. It is important to characterize the type of MI as well as the extent of the infarct, residual LV function, and the severity of CAD and other risk factors, rather than merely making a diagnosis of MI. The information conveyed about the patient's prognosis and ability to work requires more than just the mere statement that the patient has suffered an MI. The many additional factors just mentioned are also 
required so that appropriate social, family, and employment decisions can be made. A number of risk scores have been developed to predict the prognosis after MI. The classification of the various other prognostic entities associated with MI should lead to a reconsideration of the clinical coding entities currently employed for patients with the myriad conditions that can lead to myocardial necrosis, with consequent elevation of biomarker values.

It should be appreciated that the current modification of the definition of MI may be associated with consequences for the patients and their families in respect of psychological status, life insurance, professional career, as well as driving and pilots' licences. The diagnosis is associated also with societal implications as to diagnosis-related coding, hospital reimbursement, public health statistics, sick leave, and disability attestation. In order to meet this challenge, physicians must be adequately informed of the altered diagnostic criteria. Educational materials will need to be created and treatment guidelines must be appropriately adapted. Professional societies and health care planners should take steps to facilitate the rapid dissemination of the revised definition to physicians, other health care professionals, administrators, and the general public.

\section{Global perspectives of the definition of myocardial infarction}

Cardiovascular disease is a global health problem. Understanding the burden and effects of CAD in populations is of critical importance. Changing clinical definitions, criteria and biomarkers add challenges to our understanding and ability to improve the health of the public. The definition of MI for clinicians has important and immediate therapeutic implications. For epidemiologists, the data are usually retrospective, so consistent case definitions are critical for comparisons and trend analysis. The standards described in this report are suitable for epidemiology studies. However, to analyze trends over time, it is important to have consistent definitions and to quantify adjustments when biomarkers or other diagnostic criteria change. ${ }^{101}$ For example, the advent of cTn dramatically increased the number of diagnosable MIs for epidemiologists. ${ }^{3,102}$

In countries with limited economic resources, cardiac biomarkers and imaging techniques may not be available except in a few centers, and even the option of ECG recordings may be lacking. In these surroundings, the WHO states that biomarker tests or other high-cost diagnostic testing are unfit for use as compulsory diagnostic criteria. ${ }^{3}$ The WHO recommends the use of the ESC/ACCF/AHA/WHF Universal MI Definition in settings without resource constraints, but recommends more flexible standards in resource-constrained locations. ${ }^{3}$

Cultural, financial, structural, and organizational problems in the different countries of the world in the diagnosis and therapy of acute MI will require ongoing investigation. It is essential that the gap between therapeutic and diagnostic advances be addressed in this expanding area of cardiovascular disease.
1. The Joint European Society of Cardiology/ American College of Cardiology Committee. Myocardial infarction redefined-a consensus document of the Joint European Society of Cardiology/American College of Cardiology Committee for the redefinition of myocardial infarction. Eur. Heart J. 21, 1502-1513; J. Am. Coll. Cardiol. 36, 959-969 (2000).

2. Thygesen, K., Alpert, J. S., White, H. D., Joint ESC/ACCF/AHA/WHF Task Force for the Redefinition of Myocardial Infarction. Universal definition of myocardial infarction. Eur. Heart J. 28, 2525-2538; Circulation 116, 2634-2653; J. Am. Coll. Cardiol. 50, 2173-2195 (2007).

3. Mendis, S. et al. on behalf of the participating experts of the WHO consultation for revision of WHO definition of myocardial infarction. World Health Organization definition of myocardial infarction: 2008-09 revision. Int. J. Epidemiol. 40, 139-146 (2011).

4. Jennings, R. B. \& Ganote, C. E. Structural changes in myocardium during acute ischemia. Circ. Res. 35 (Suppl. 3), 156-172 (1974).

5. Jaffe, A. S., Babuin, L. \& Apple, F. S. Biomarkers in acute cardiac disease. J. Am. Coll. Cardiol. 48, 1-11 (2006).

6. White, H. D. Pathobiology of troponin elevations. J. Am. Coll. Cardiol. 57, 2406-2408 (2011).

7. Jaffe, A. S. Chasing troponin: how low can you go if you can see the rise? J. Am. Coll. Cardiol. 48 1763-1764 (2006).

8. Apple, F. S., Jesse, R. L., Newby, L. K., Wu, A. H. B. \& Christenson, R. H. National Academy of Clinical Biochemistry and IFCC Committee for Standardization of Markers Cardiac Damage Laboratory Medicine Practice Guidelines: analytical issues for biochemical markers of acute coronary syndromes. Circulation 115, e352-e355 (2007).

9. Morrow, D. A. et al. National Academy of Clinical Biochemistry Laboratory Medicine Practice Guidelines: clinical characteristics and utilization of biochemical markers of acute coronary syndromes. Circulation 115, e356-e375 (2007).

10. Thygesen, $K$. et al. Recommendations for the use of cardiac troponin measurement in acute cardiac care. Eur. Heart J. 31, 2197-2204 (2010).

11. Thygesen, K. et al. How to use high-sensitivity cardiac troponins in acute cardiac care. Eur. Heart J. http://dx.doi.org/10.1093/eurheartj/ehs154.

12. Apple, F. S., Collinson, P. O. \& IFCC Task Force on Clinical Applications of Cardiac Biomarkers. Analytical characteristics of high-sensitivity cardiac troponin assays. Clin. Chem. 58, 54-61 (2012).

13. Jaffe, A. S., Apple, F. S., Morrow, D. A., Lindahl, B. \& Katus, H. A. Being rational about (im)precision: a statement from the Biochemistry Subcommittee of the Joint European Society of Cardiology/American College of Cardiology Foundation/American Heart Association/World Heart Federation Task Force for the definition of myocardial infarction. Clin. Chem. 56, 941-943 (2010).

14. MacRae, A. R. et al. Assessing the requirement for the six-hour interval between specimens in the American Heart Association classification of myocardial infarction in epidemiology and clinical research studies. Clin. Chem. 52, 812-818 (2006).

15. de Lemos, J. A. et al. Association of troponin T detected with a highly sensitive assay and cardiac structure and mortality risk in the general population. JAMA 304, 2503-2512 (2010).

16. Omland, T. et al. A sensitive cardiac troponin $\mathrm{T}$ assay in stable coronary artery disease. N. Engl. J. Med. 361, 2538-2547 (2009).
17. Mills, N. L. et al. Implementation of a sensitive troponin I assay and risk of recurrent myocardial infarction and death in patients with suspected acute coronary syndrome. JAMA 305 , 1210-1216 (2011).

18. Saunders, J. T. et al. Cardiac troponin T measured by a highly sensitive assay predicts coronary heart disease, heart failure, and mortality in the atherosclerosis risk in communities study. Circulation 123, 1367-1376 (2011).

19. Kavsak, P. A., Xu, L., Yusuf, S. \& McQueen, M. J. High-sensitivity cardiac troponin I measurement for risk stratification in a stable high-risk population. Clin. Chem. 57, 1146-1153 (2011).

20. Apple, F. S., Simpson, P. A. \& Murakami, M. M. Defining the serum $99^{\text {th }}$ percentile in a normal reference population measured by a highsensitivity cardiac troponin I assay. Clin. Biochem. 43, 1034-1036 (2010).

21. Giannitsis, E. et al. Analytical validation of a highsensitivity cardiac troponin T assay. Clin. Chem. 56, 254-261 (2010).

22. Apple, F. S., Quist, H. E., Doyle, P. J., Otto, A. P. \& Murakami, M. M. Plasma $99^{\text {th }}$ percentile reference limits for cardiac troponin and creatine kinase MB mass for use with European Society of Cardiology/American College of Cardiology consensus recommendations. Clin. Chem. 49 , 1331-1336 (2003).

23. Roe, M. T. et al. Clinical and therapeutic profile of patients presenting with acute coronary syndromes who do not have significant coronary artery disease. The Platelet glycoprotein Ilb/IIla in Unstable angina: Receptor Suppression Using Integrilin Therapy (PURSUIT) trial investigators. Circulation 102, 1101-1106 (2000). 
24. Bugiardini, R., Manfrini, O. \& De Ferrari, G. M. Unanswered questions for management of acute coronary syndrome: risk stratification of patients with minimal disease or normal findings on coronary angiography. Arch. Intern. Med. 166, 1391-1395 (2006).

25. Reynolds, H. R. et al. Mechanisms of myocardial infarction in women without angiographically obstructive coronary artery disease. Circulation 124, 1414-1425 (2011).

26. Bertrand, M. E. et al. Frequency of provoked coronary arterial spasm in 1089 consecutive patients undergoing coronary arteriography. Circulation 65, 1299-1306 (1982).

27. Suwaidi, J. A. et al. Long-term follow-up of patients with mild coronary artery disease and endothelial dysfunction. Circulation 101, 948-954 (2000).

28. Bugiardini, R., Manfrini, O., Pizzi, C., Fontana, F. \& Morgagni, G. Endothelial function predicts future development of coronary artery disease: a study on women with chest pain and normal angiograms. Circulation 109, 2518-2523 (2004).

29. Harris, B. M., Nageh, T., Marsden, J. T., Thomas, M. R. \& Sherwood, R. A. Comparison of cardiac troponin $\mathrm{T}$ and $\mathrm{I}$ and $\mathrm{CK}-\mathrm{MB}$ for the detection of minor myocardial damage during interventional cardiac procedures. Ann. Clin. Biochem. 37, 764-769 (2000).

30. Januzzi, J. L. et al. A comparison of cardiac troponin T and creatine kinase-MB for patient evaluation after cardiac surgery. J.Am. Coll. Cardiol. 39, 1518-1523 (2002).

31. Holmvang, L. et al. Use of biochemical markers of infarction for diagnosing perioperative myocardial infarction and early graft occlusion after coronary artery bypass surgery. Chest 121 , 103-111 (2002).

32. Miller, W. L., Garratt, K. N., Burritt, M. F., Reeder, G. S. \& Jaffe, A. S. Timing of peak troponin T and creatine kinase-MB elevations after percutaneous coronary intervention. Chest 25, 275-280 (2004).

33. Lansky, A. J. \& Stone, G. W. Periprocedural myocardial infarction: prevalence, prognosis, and prevention. Circ. Cardiovasc. Interv. 3, 602-610 (2010).

34. Cavallini, C. et al. Prognostic value of isolated troponin I elevation after percutaneous coronary intervention. Circ. Cardiovasc. Interv. 3, 431-435 (2010).

35. Prasad, A. Jr et al. Significance of periprocedural myonecrosis on outcomes following percutaneous coronary intervention. Circ. Cardiovasc. Interv. 1, 10-19 (2008).

36. Zimetbaum, P. J. \& Josephson, M. E. Use of the electrocardiogram in acute myocardial infarction. N. Engl. J. Med. 348, 933-940 (2003).

37. Wang, K., Asinger, R. W. \& Marriott, H. J. ST-segment elevation in conditions other than acute myocardial infarction. N. Engl. J. Med. 349, 2128-2135 (2003).

38. Mcfarlane, P. W. Age, sex, and the ST amplitude in health and disease. J. Electrocardiol. 34 (Suppl.), S35-S41 (2001).

39. Zimetbaum, P. J., Krishnan, S., Gold, A., Carrozza, J. P. $2^{\text {nd }}$ \& Josephson, M. E. Usefulness of ST-segment elevation in lead III exceeding that of lead II for identifying the location of the totally occluded coronary artery in inferior wall myocardial infarction. Am. J. Cardiol. 81, 918-919 (1998).

40. Engelen, D. J. et al. Value of the electrocardiogram in localizing the occlusion site in the left anterior descending coronary artery in acute anterior myocardial infarction. J.Am. Coll. Cardiol. 34, 389-395 (1999).
41. Matetzky, S. et al. Acute myocardial infarction with isolated ST-segment elevation in posterior chest leads $\mathrm{V}_{7}-\mathrm{V}_{9}$. Hidden ST-segment elevations revealing acute posterior infarction. J. Am. Coll. Cardiol. 34, 748-753 (1999).

42. Lopez-Sendon, J., Coma-Canella, I., Alcasena, S., Seoane, J. \& Gamallo, C. Electrocardiographic findings in acute right ventricular infarction: sensitivity and specificity of electrocardiographic alterations in right precordial leads $\mathrm{V}_{4} \mathrm{R}, \mathrm{V}_{3} \mathrm{R}, \mathrm{V}_{1}$, $\mathrm{V}_{2}$ and $\mathrm{V}_{3}$. J. Am. Coll. Cardiol. 6, 1273-1279 (1985).

43. Bayés de Luna, A. et al. A new terminology for the left ventricular walls and for the location of myocardial infarcts that present Q wave based on the standard of cardiac magnetic resonance imaging. A statement for healthcare professionals from a Committee appointed by the International Society for Holter and Noninvasive Electrocardiography. Circulation 114, 1755-1760 (2006).

44. Sgarbossa, E. B. et al. Electrocardiographic diagnosis of evolving acute myocardial infarction in the presence of left bundle branch block. N. Engl. J. Med. 334, 481-487 (1996).

45. Jain, S. et al. Utility of left bundle branch block as a diagnostic criterion for acute myocardial infarction. Am. J. Cardiol. 107, 1111-1116 (2011).

46. Savage, R. M., Wagner, G. S., Ideker, R. E., Podolsky, S. A. \& Hackel, D. B. Correlation of postmortem anatomic findings with electrocardiographic changes in patients with myocardial infarction: retrospective study of patients with typical anterior and posterior infarcts. Circulation 55, 279-285 (1977).

47. Horan, L. G., Flowers, N. C. \& Johnson, J. C. Significance of the diagnostic $Q$ wave of myocardial infarction. Circulation 43, 428-436 (1971).

48. Chaitman, B. R. et al. The Bypass Angioplasty Revascularization Investigation 2 Diabetes randomized trial of different treatment strategies in type 2 diabetes mellitus with stable ischemic heart disease: impact of treatment strategy on cardiac mortality and myocardial infarction. Circulation 120, 2529-2540 (2009).

49. Burgess, D. C. et al. Incidence and predictors of silent myocardial infarction in type 2 diabetes and the effect of fenofibrate: an analysis from the Fenofibrate Intervention and Event Lowering in Diabetes (FIELD) study. Eur. Heart J. 31, 92-99 (2010).

50. Sheifer, S. E., Manolio, T. A. \& Gersh, B. J. Unrecognized myocardial infarction. Ann. Intern. Med. 135, 801-811 (2001).

51. Toma, M. et al. Does silent myocardial infarction add prognostic value in ST-elevation myocardial infarction? Insights from the Assessment of Pexelizumab in Acute Myocardial Infarction (APEX-AMI) trial. Am. Heart J. 160, 671-677 (2010).

52. Stillman, A. E. et al. Assessment of acute myocardial infarction: current status and recommendations from the North American Society for Cardiovascular Imaging and the European Society of Cardiac Radiology. Int. J. Cardiovasc. Imaging 27, 7-24 (2011).

53. Flachskampf, F. A. et al. Cardiac imaging after myocardial infarction. Eur. Heart J. 32, 272-283 (2011).

54. Kaul, S. et al. A suggested roadmap for cardiovascular ultrasound research for the future. J. Am. Soc. Echocardiogr. 24, 455-464 (2011).

55. Carrio, I., Cowie, M. R., Yamazaki, J., Udelson, J. \& Camici, P. G. Cardiac sympathetic imaging with mIBG in heart failure. JACC Imaging 3, 92-100 (2010).
56. Nahrendorf, M. et al. Multimodality cardiovascular molecular imaging, part II. Circ. Cardiovasc. Imaging 2, 56-70 (2009).

57. Kramer, C. M., Sinusas, A. J., Sosnovik, D. E., French, B. A. \& Bengel, F. M. Multimodality imaging of myocardial injury and remodeling. J. Nucl. Med. 51 (Suppl. 1), 107S-121S (2010).

58. Taegtmeyer, H. Tracing cardiac metabolism in vivo: one substrate at a time. J. Nucl. Med. 51 (Suppl. 1), 80S-87S (2010).

59. Kim, H. W., Faraneh-Far, A. \& Kim, R. J. Cardiovascular magnetic resonance in patients with myocardial infarction. J. Am. Coll. Cardiol. 55, 1-16 (2010).

60. Beek, A. M. \& van Rossum, A. C. Cardiovascular magnetic resonance imaging in patients with acute myocardial infarction. Heart 96, 237-243 (2010).

61. Assomull, R. G. et al. The role of cardiovascular magnetic resonance in patients presenting with chest pain, raised troponin, and unobstructed coronary arteries. Eur. Heart J. 28, 1242-1249 (2007).

62. Schuleri, K. H., George, R. T. \& Lardo, A. C. Assessment of coronary blood flow with computed tomography and magnetic resonance imaging. J. Nucl. Cardiol. 17, 582-590 (2010).

63. Amsterdam, E. A. et al. Testing of low-risk patients presenting to the emergency department with chest pain. Circulation 122, 1756-1776 (2010).

64. Gibbons, R. J., Valeti, U. S., Araoz, P. A. \& Jaffe, A. S. The quantification of infarct size. J. Am. Coll. Cardiol. 44, 1533-1542 (2004).

65. Herrman, J. Peri-procedural myocardial injury: 2005 update. Eur. Heart J. 26, 2493-2519 (2005).

66. Selvanayagam, J. B. et al. Troponin elevation after percutaneous coronary intervention directly represents the extent of irreversible myocardial injury: insights from cardiovascular magnetic resonance imaging. Circulation 111 , 1027-1032 (2005).

67. Gustavsson, C. G., Hansen, O. \& Frennby, B. Troponin must be measured before and after PCl to diagnose procedure-related myocardial injury. Scand. Cardiovasc. J. 38, 75-79 (2004).

68. Miller, W. L. et al. Baseline troponin level: key to understanding the importance of post-PCI troponin elevations. Eur. Heart J. 27, 1061-1069 (2006).

69. Califf, R. M. et al. Myonecrosis after revascularization procedures. J. Am. Coll. Cardiol. 31, 241-251 (1998).

70. White, H. D. The prequel. Defining prognostically important criteria in the periprocedural $\mathrm{PCl}$ troponin saga. Circ. Cardiovasc. Interv. 5, 142-145 (2012)

71. Jaffe, A. S., Apple, F. S., Lindahl, B., Mueller, C. \& Katus, H. A. Why all the struggle about CK-MB and PCI? Eur. Heart J. 33, 1046-1048 (2012).

72. Damman, P. et al. Long-term cardiovascular mortality after procedure-related or spontaneous myocardial infarction in patients with non-STsegment elevation acute coronary syndrome: a collaborative analysis of individual patient data from the FRISC II, ICTUS, and RITA-3 trials (FIR). Circulation 125, 568-576 (2012).

73. Bonaca, M. P. et al. American College of Cardiology/American Heart Association/ European Society of Cardiology/World Heart Federation Universal Definition of Myocardial Infarction Classification System and the risk of cardiovascular death: observations from the TRITON-TIMI 38 trial (Trial to Assess Improvement in Therapeutic Outcomes by Optimizing Platelet Inhibition With PrasugrelThrombolysis in Myocardial Infarction 38). Circulation 125, 577-583 (2012). 
74. Cutlip, D. E. et al. Clinical end points in coronary stent trials: a case for standardized definitions. Circulation 115, 2344-2351 (2007).

75. Benoit, M. O., Paris, M., Silleran, J., Fiemeyer, A. \& Moatti, N. Cardiac troponin I: its contribution to the diagnosis of perioperative myocardial infarction and various complications of cardiac surgery. Crit. Care Med. 29, 1880-1886 (2001).

76. Kovacevic, R. et al. Troponin T levels in detection of perioperative myocardial infarction after coronary artery bypass surgery. Clin. Lab. 50, 437-445 (2004)

77. Noora, J., Ricci, C., Hastings, D., Hills, S. \& Cybulsky, I. Determination of troponin I release after CABG surgery. J. Card. Surg. 20, 129-135 (2005).

78. Selvanayagam, J. B. et al. Relationship of irreversible myocardial injury to troponin I and creatine kinase-MB elevation after coronary artery bypass surgery: insights from cardiovascular magnetic resonance imaging. J. Am. Coll. Cardiol. 45, 629-631 (2005).

79. Costa, M. A. et al. Incidence, predictors, and significance of abnormal cardiac enzyme rise in patients treated with bypass surgery in the Arterial Revascularization Therapies Study (ARTS). Circulation 104, 2689-2693 (2001).

80. Klatte, K. et al. Increased mortality after coronary artery bypass graft surgery is associated with increased levels of postoperative creatine kinase-myocardial band isoenzyme release. J. Am. Coll. Cardiol. 38 , 1070-1077 (2001).

81. Brener, S. J., Lytle, B. W., Schneider, J. P., Ellis, S. G. \& Topol, E. J. Association between CK-MB elevation after percutaneous or surgical revascularization and three-year mortality. J. Am. Coll. Cardiol. 40, 1961-1967 (2002).

82. Domanski, M. et al. Association of myocardial enzyme elevation and survival following coronary artery bypass graft surgery. JAMA 305, 585-589 (2011).

83. Croal, B. L. et al. Relationship between postoperative cardiac troponin I levels and outcome of cardiac surgery. Circulation 114, 1468-1475 (2006).

84. Selvanayagam, J. B. et al. Effects of off-pump versus on-pump coronary surgery on reversible and irreversible myocardial injury: a randomized trial using cardiovascular magnetic resonance imaging and biochemical markers. Circulation 109, 345-350 (2004).

85. Leon, M. B. et al. Standardized endpoint definitions for transcatheter aortic valve implantation clinical trials: a consensus report from the Valve Academic Research Consortium.
Eur. Heart J. 32, 205-217; J. Am. Coll. Cardiol. 57, 253-269 (2011).

86. Devereaux, P. J. et al. Characteristics and shortterm prognosis of perioperative myocardial infarction in patients undergoing noncardiac surgery: a cohort study. Ann. Intern. Med. 154, 523-528 (2011).

87. The Vascular Events in Noncardiac Surgery Patients Cohort Evaluation (VISION) Study Investigators. Association between postoperative troponin levels and 30-day mortality among patients undergoing noncardiac surgery. JAMA 307, 2295-2304 (2012).

88. Kavsak, P. A. et al. High sensitivity troponin T concentrations in patients undergoing noncardiac surgery: a prospective cohort study. Clin. Biochem. 44, 1021-1024 (2011).

89. Fleisher, L. A., Nelson, A. H. \& Rosenbaum, S. H. Postoperative myocardial ischemia: etiology of cardiac morbidity or manifestation of underlying disease? J. Clin. Anesth. 7, 97-102 (1995).

90. Landesberg, G. et al. Cardiac troponin after major vascular surgery: the role of perioperative ischemia, preoperative thallium scanning, and coronary revascularization. J. Am. Coll. Cardiol. 44, 569-575 (2004).

91. Cohen, M. C. \& Aretz, T. H. Histological analysis of coronary artery lesions in fatal postoperative myocardial infarction. Cardiovasc. Pathol. 8, 133-139 (1999).

92. Guest, T. M. et al. Myocardial injury in critically ill medical patients: a surprisingly frequent complication. JAMA 273, 1945-1949 (1995).

93. Babuin, L. et al. Elevated cardiac troponin is an independent risk factor for short- and long-term mortality in medical intensive care unit patients. Crit. Care Med. 36, 759-765 (2008)

94. Landesberg, G. et al. Myocardial ischemia, cardiac troponin, and long-term survival of highcardiac risk critically ill intensive care unit patients. Crit. Care Med. 33, 1281-1287 (2005).

95. Thygesen, K., Alpert, J. S., Jaffe, A. S. \& White, H. D. Diagnostic application of the universal definition of myocardial infarction in the intensive care unit. Curr. Opin. Crit. Care 14 543-548 (2008).

96. Kociol, R. D. et al. Troponin elevation in heart failure prevalence, mechanisms, and clinical implications. J. Am. Coll. Cardiol. 56, 1071-1078 (2010).

97. Januzzi, J. L. Jr, Filippatos, G., Nieminen, M. \& Gheorghiade, M. Troponin elevation in patients with heart failure: on behalf of the Third Universal Task Force for the Definition of Myocardial Infarction Global Task Force: Heart Failure Section. Eur. Heart J. http://dx.doi.org/ 10.1093/eurheartj/ehs191.
98. Miller, W. L., Hartman, K. A., Burritt, M. F., Grill, D. E. \& Jaffe, A. S. Profiles of seria changes in cardiac troponin T concentrations and outcome in ambulatory patients with chronic heart failure. J. Am. Coll. Cardiol. 54, 1715-1721 (2009).

99. Dangas, G. D. et al. In-stent restenosis in the drug-eluting era. J. Am. Coll. Cardiol. 56, 1897-1907 (2010).

100. White, H. D. et al. Reinfarction after percutaneous coronary intervention or medica management using the universal definition in patients with total occlusion after myocardial infarction: results from long-term follow-up of the Occluded Artery Trial (OAT) cohort. Am. Heart J. 163, 563-571 (2012).

101. Rosamond, W. et al. Twenty-two year trends in incidence of myocardial infarction, CHD mortality, and case-fatality in four US communities, 1987 to 2008. Circulation 125, 1848-1857 (2012).

102. Luepker, R., Duval, S., Jacobs, D., Smith, L. $\&$ Berger, A. The effect of changing diagnostic algorithms on acute myocardial infarction rates. Ann. Epidemiol. 21, 824-829 (2011).

\section{Acknowledgments}

ESC Committee for Practice Guidelines (CPG): Jeroen J. Bax, (CPG Chairperson) (Netherlands), Helmut Baumgartner (Germany), Claudio Ceconi (Italy), Veronica Dean (France), Christi Deaton (UK), Robert Fagard (Belgium), Christian Funck-Brentano (France), David Hasdai (Israel), Arno Hoes (Netherlands), Paulus Kirchhof (Germany/UK), Juhani Knuuti (Finland), Philippe Kolh (Belgium), Theresa McDonagh (UK), Cyril Moulin (France), Bogdan A. Popescu (Romania), Željko Reiner (Croatia), Udo Sechtem (Germany), Per Anton Sirnes (Norway), Michal Tendera (Poland), Adam Torbicki (Poland), Alec Vahanian (France), Stephan Windecker (Switzerland). Document Reviewers: Joao Morais, (CPG Review Coordinator) (Portugal), Carlos Aguiar (Portugal), Wael Almahmeed (United Arab Emirates), David O. Arnar (Iceland), Fabio Barili (Italy), Kenneth D. Bloch (USA), Ann F. Bolger (USA), Hans Erik Bøtker (Denmark), Biykem Bozkurt (USA), Raffaele Bugiardini (Italy), Christopher Cannon (USA), James de Lemos (USA), Franz R. Eberli (Switzerland), Edgardo Escobar (Chile), Mark Hlatky (USA), Stefan James (Sweden), Karl B. Kern (USA), David J. Moliterno (USA), Christian Mueller (Switzerland), Aleksandar N. Neskovic (Serbia), Burkert Mathias Pieske (Austria), Steven P. Schulman (USA), Robert F. Storey (UK), Kathryn A. Taubert (Switzerland), Pascal Vranckx (Belgium), Daniel R. Wagner (Luxembourg).

We are very grateful to the dedicated staff of the Practice Guidelines Department of the ESC. 